\title{
Propagation and ion dynamics of waves in amplitude modulated helicon plasmas
}

\author{
A. Stark* , O. Grulke*,† and T. Klinger*,† \\ ${ }^{*}$ Max-Planck Institute for Plasma Physics, EURATOM Association, Greifswald, Germany \\ ${ }^{\dagger}$ Ernst-Moritz-Arndt Universität, Greifswald, Germany
}

\begin{abstract}
A helicon source is driven with amplitude modulated RF at modulation frequencies near the ion cyclotron frequency in the VINETA device. In response to the modulation propagating electromagnetic waves are found, whose dispersion differs significantly from linear wave dispersion theory. This indicates that the observed waves are generated by non-linear wave coupling of the helicon waves with a second strongly damped or evanescent wave. Laser induced fluorescence measurements reveal a local increase of the ion temperature at the minimum of the modulation drive.
\end{abstract}

Keywords: three-wave-coupling,helicon waves,modulation,ion kinetics, laser induced fluorescence PACS: 52.35.-g, 52.70.-m

\section{Introduction}

The concept of three wave coupling is well established in the current literature [1]. Closely related to that is the modulational instability, which results from the coupling of waves modes of very different time and space scales [2]. Conversely, a modulation of a high frequency wave can, due to non-linear wave coupling, launch low frequency waves with non-linear dispersion behaviour. Low frequency waves created by non-linear processes are of interest to explain observations made in geophysical and space plasmas [3]. Of particular importance here are the dynamics and kinetics of the ions.

Laser induced fluorescence (LIF) [4] has become a standard diagnostic tool for ion temperature and ion drift measurements in plasmas. LIF is non-invasive and provides a high spatial and a high energy resolution. With the recent development of advanced laser systems LIF is more and more used to detect also perturbations of the ion velocity distribution function (IVDF) and to get detailed information about the ion dynamics [5].

This paper reports on amplitude modulation of a RF helicon source. Propagating electromagnetic waves are detected, were the phase velocity agrees well with the Alfvén velocity. Hence the ion dynamics are investigated separately by using LIF. The paper is structured as follows: The first section describes the device and the set of diagnostics. In the second section results from dispersion and LIF measurements are presented. A short summary and conclusion is given at the end of the paper.

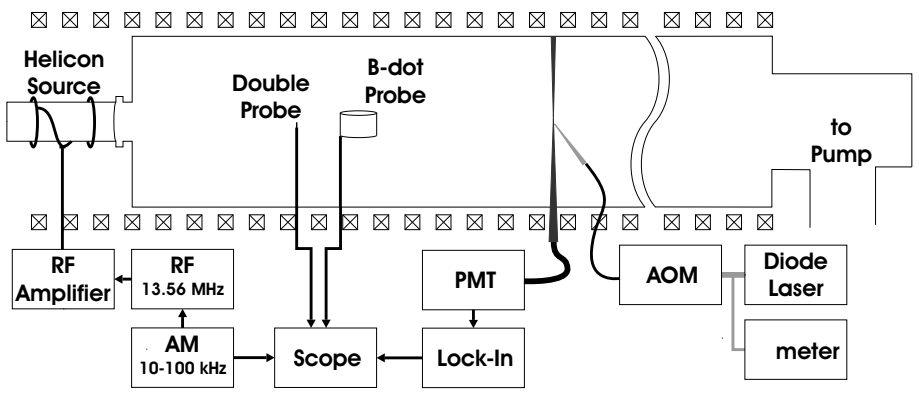

FIGURE 1. Schematic diagram of the VINETA device and used diagnostics. Shown is the principle setup of the LIF diagnostics. Movable double-tip $E$-field and $\dot{B}$-probes are used for dispersion measurements. 


\section{Experimental arrangement}

The experiments were done in the magnetized linear device VINETA [6]. Figure 1 shows a schematic diagram of the experimental setup. Four identical modules form the $4.5 \mathrm{~m}$ long vacuum chamber, which is immersed in a set of 36 magnetic field coils $\left(B_{0} \leq 100 \mathrm{mT}\right)$. The helicon plasma source consists of a $m=1$ helical antenna placed around a glass tube extension at one end of the device. The antenna is driven with RF frequency at $13.56 \mathrm{MHz}$ and RF power of $P_{r f} \leq 6 \mathrm{~kW}$. In helicon wave sustained mode, high plasma densities with $n \approx 1 \cdot 10^{19} \mathrm{~m}^{-3}$ (monitored by a $160 \mathrm{GHz}$ interferometer) are achieved. Langmuir probe measurements show that the electron temperature is in the range of $1-5 \mathrm{eV}$. Argon is typically used as filling gas at pressures of $5 \cdot 10^{-2}-5 \cdot 10^{-1} \mathrm{~Pa}$.

The modular design of the RF amplifier unit allows a simple way of modulating the RF amplitude by using a arbitrary function generator. Both the modulation depth $A_{m}$ and the modulation frequency $f_{m}$ are varied. The modulation depth was chosen between $A_{m}=10 \ldots 100 \%$ and the modulation frequency was varied around the ion cyclotron frequency $f_{m}=10 \ldots 100 \mathrm{kHz}$. The plasma response and electromagnetic fluctuations are measured by magnetic and electrostatic probes [7]. A floating double probe is used to measure the perturbed electric field. An uncompensated negatively biased Langmuir probe is used to measure the ion saturation current. Time-averaged electron temperatures and plasma densities are obtained by evaluation of probe characteristics. Magnetic field fluctuations are measured with $\dot{B}$-probes, which consists of a coil with $2 \mathrm{~cm}$ diameter and 1600 windings embedded in a copper heat shield and a boron nitride body. The double probe is oriented to measure radial electric fields and the $\dot{B}$-probe to measure oscillations perpendicular to the ambient magnetic field. The probes are mounted on a positioning system, that allows radial and axial movement of the probes.

The IVDF is measured by means of LIF. Here, a $60 \mathrm{~mW} \mathrm{cw}$ diode laser, tunable over $20 \mathrm{pm}$, is used to pump the Argon II $3 d{ }^{4} F_{7 / 2}-4 p{ }^{4} D_{5 / 2}$ transition at $668.614 \mathrm{~nm}$. The fluorescence radiation of the $4 p{ }^{4} D_{5 / 2}-4 s{ }^{4} P_{3 / 2}$ transition at $442.72 \mathrm{~nm}$ is observed by a photomultiplier tube (PMT) after passing a $1 \mathrm{~nm}$ bandwidth interference filter. Spontaneous emission is discriminated from laser induced emissions by chopping the laser light and using a LockIn technique. Mechanical choppers are technically limited to frequencies below $\approx 10 \mathrm{kHz}$. This limitation has two drawbacks: Firstly, a low chopper frequency often results in a poor signal-to-noise ratio, due to low frequency noise as found in the helicon mode. Secondly, unless the chopper frequency is of the order of the perturbation drive, the twofold discrimination process (LIF and perturbation) becomes more difficult. Instead of using a Lock-In amplifier, high resolution data acquisition and extensive off-line spectral analysis is made [8]. The use of acousto-optical modulators (AOM) yield high chopper frequencies of several $\mathrm{MHz}$ at high transmission rates and high contrast ratios [9]. All active diagnostic parts together, the AOM, the PMT with amplifier and the Lock-In amplifier, permit time resolved measurements up to $10 \mu \mathrm{s}$ time resolution.

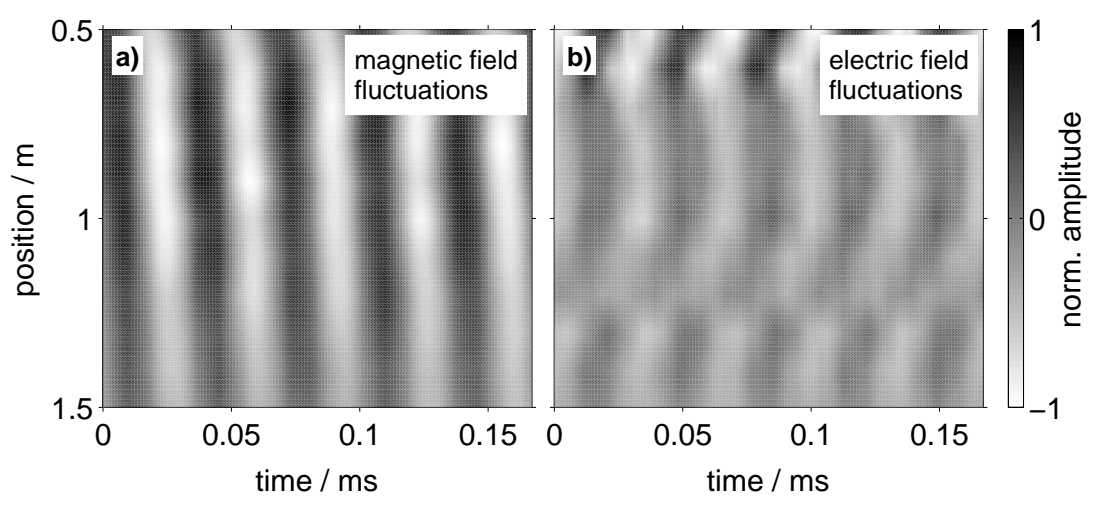

FIGURE 2. Spatio-temporal measurement of the magnetic (a) and electric (b) field fluctuations at a modulation depth of $A_{m}=10 \%$ and modulation frequency of $f_{m}=30 \mathrm{kHz}$. The respective normalized amplitude is plotted gray-scale coded. 

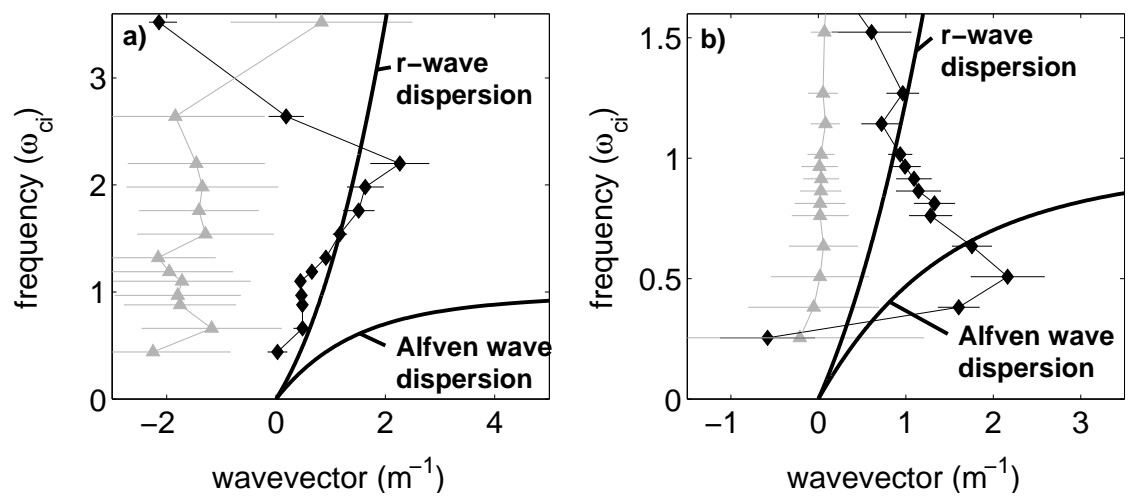

FIGURE 3. Dispersion measurements of the propagating magnetic field fluctuations (black) and the electric field fluctuations (gray): (a) dispersion at $60 \mathrm{mT}$ magnetic field $\left(\omega_{c i}=380 \mathrm{kHz}\right)$, (b) $100 \mathrm{mT}$ magnetic field $\left(\omega_{c i}=230 \mathrm{kHz}\right)$. Included in the graphs are the theoretical linear dispersion relations of Alfvén waves and r-waves.

\section{Experimental results}

The global plasma response to the modulation of the RF-amplitude is seen in the interferometer signal as a dip in the plasma density. However, at low modulation depth of $A_{m}=10 \%$ no temporal variation of the plasma density is seen. Instead, fluctuations are observed in both the double probe and $\dot{B}$-probe signal.

In Fig. 2 the spatio-temporal amplitude evolution of the magnetic and the electric field fluctuations are plotted grayscale coded. The magnetic field (Fig. 2(a)) exhibits a regular striped pattern of a single propagating electromagnetic wave, in which the tilt of the stripes yields the phase velocity and the evolution of the amplitude the damping length. The wave is of Alfvénic nature with a phase velocity $v_{p h}=2 \cdot 10^{5} \mathrm{~ms}^{-1}$ close to the Alfvén velocity. A more complex picture is found for the electric field (Fig. 2(b)). The pattern exhibits a spatial and temporal modulation, i.e the propagation direction changes depending on the respective axial position. This irregular pattern arises presumably from two superimposed waves of different wavelength and amplitude. However, as the magnetic fluctuation pattern indicates, the second wave is of pure electrostatic nature.

For different modulation frequencies the low frequency wave dispersion is recorded: Fig. 3 shows the result of two dispersion measurements at different magnetic fields of $60 \mathrm{mT}$ and $100 \mathrm{mT}$, respectively. The phase velocity of both the magnetic and the electric component were measured in the range of $f_{m}=0.5-3.5 \omega_{c i} / 2 \pi$ and $f_{m}=0.25-1.5 \omega_{c i} / 2 \pi$, where $\omega_{c i}$ denotes the ion cyclotron frequency. The respective wave dispersions are plotted as frequency $\omega$ vs. wavevector perpendicular to the ambient magnetic field $k_{\perp}$. Due to the superposition of different propagating waves, the electric component exhibits much bigger errorbars and the dispersion differs significantly from that of the magnetic component. At low magnetic field the dispersion follows a $\mathrm{r}$-wave dispersion, even at frequencies below the ion cyclotron frequency where Alfvén waves can propagate. At the higher magnetic field an anomalous dispersion is found, which is characterized by a decreasing phase velocity with increasing wavevector (backward waves [10]).

The ion kinetic response of the velocity component perpendicular to $B_{0}$ to the low frequency wave as measured with LIF is shown in Fig. 4. The measurements of the time averaged IVDF with and without amplitude modulation shows only differences in the intensity, not in the width of the IVDF (c.f. Fig. 4(a)). To emphasize the similarity of both measurements, the LIF intensity is normalized to the maximum value. The ion temperature is found to be constant at $T_{i}=0.34 \mathrm{eV}$ and the drift is almost zero with $v_{d}=50 \mathrm{~m} / \mathrm{s}^{-1}$. Even at modulation depth of $A_{m}=100 \%$ no modification of the IVDF is found. The time evolution of the ion response is obtained by phase resolved measurements in the following way: Time series of the LIF signal were recorded and averaged phase locked over several 100 modulation drive periods to increase the statistical significance. The qualitative behaviour of the phase resolved IVDF is seen for all modulation depth, but is strongest at maximum modulation. The phase resolved IVDF, normalized to the maximum value, is plotted gray-scale coded in Fig. 4(b). The phase reference is indicated in Fig. 4(c) (dashed line). Here, the modulation depth is $A_{m}=100 \%$ and the frequency was set to $f_{\text {mod }}=30 \mathrm{kHz}$. At the maximum and minimum of the modulation drive local maxima of the LIF intensity are observed. Gaussians fitted to each IVDF (black contours) yield the temporal evolution of the ion temperature and mean ion drift as plotted in Fig. 4(c). The ion temperature stays constant in the positive half wave of the modulation drive and varies in the negative half wave, where the maximum temperature coincides with the minimum of the modulation drive. The ion drift is found to be of the order of $0.1 c_{s}$ and 

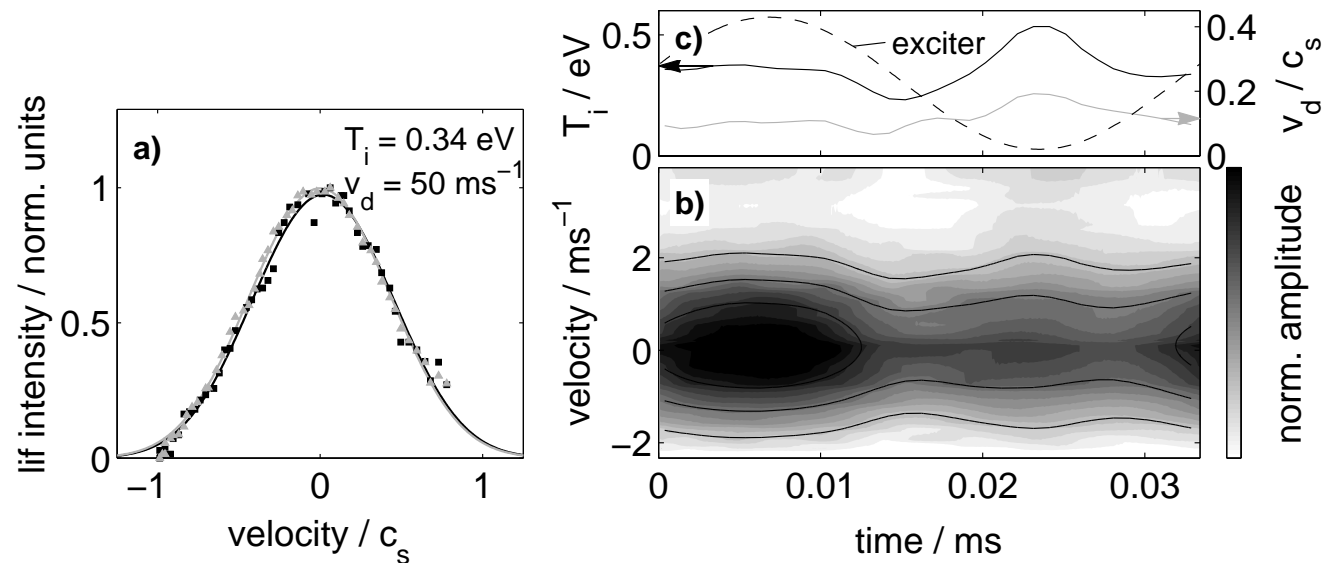

FIGURE 4. (a) Time averaged LIF intensity vs. velocity normalized to the ion sound speed $c_{s}$ with (gray) and without (black) modulation. Included are Gaussians fitted to the data (solid lines). (b) Phase resolved LIF intensity plotted in gray-scale and contours (solid lines) of the Gaussian fits. (c) Ion temperature (black) and mean drift (gray) obtained by the Gaussian fits. As a phase reference the modulation drive is included in the plot (dashed line).

varies slightly during the modulation period. The time resolved measurements reveal that the wave electric fields lead to modulation of the ion temperature, which however compensates in the time average.

\section{Summary and conclusion}

In summary measurements with electric and magnetic probes revealed propagating waves in response to amplitude modulation of a RF helicon source. The dispersion behaviour was investigated around the ion cyclotron frequency for two different magnetic field strength. It was found that the dispersion follows at low magnetic field a r-wave dispersion and changes to an anomalous dispersion for higher magnetic field. It is thus reasonable to assume that the observed low frequency waves are created by non-linear wave coupling between the helicon wave and another, probably damped or even evanescent high frequency wave. Investigations of the ion kinetics with LIF have shown a significant effect on the ion temperature only for phase resolved measurements, while the time averaged IVDF remains constant.

\section{REFERENCES}

1. V. N. Tsytovich, Lectures on Non-linear Plasma Kinetics, Springer, Berlin (Germany), 1995.

2. V. D. Shapiro, D. Ucer, and K. B. Quest, Plasma Phys. Rep., 29, 550-560 (2003).

3. A. Treumann, and W. Baumjohann, Advanced Space Plasma Physics, Imperial College Press, London (UK), 1997.

4. R. A. Stern, and J. A. Johnson, Phys. Rev. Lett., 34, 1548-1551 (1975).

5. F. Skiff, G. Bachet, and F. Doveil, Phys. Plasmas, 8, 3139-3142 (2001).

6. C. M. Franck, O. Grulke, and T. Klinger, Phys. Plasmas, 9, 3254-3258 (2002).

7. I. H. Hutchinson, Principles of Plasma Diagnostics, Cambridge University Press, Cambridge (UK), 2002.

8. A. Stark, W. Fox, J. Egedal, O. Grulke, and T. Klinger, Phys. Rev. Lett., submitted for publication (2005).

9. E. Hecht, Optics, Addison-Wesley, Boston (USA), 2001.

10. D. G. Swanson, Plasma Waves, Institute of Physics Publishing, Bristol (UK), 2003. 